\title{
Improved microRNA suppression by WPRE-linked tough decoy microRNA sponges
}

\author{
ANNE KRUSE HOLLENSEN, ${ }^{1,2}$ RUNE THOMSEN, ${ }^{2}$ RASMUS O. BAK, ${ }^{1,3}$ CHARLOTTE CHRISTIE PETERSEN, ${ }^{1}$ \\ EVA R. ERMEGAARD, ${ }^{1}$ LARS AAGAARD, ${ }^{1}$ CHRISTIAN KROUN DAMGAARD, ${ }^{2}$ and JACOB GIEHM MIKKELSEN ${ }^{1}$ \\ ${ }^{1}$ Department of Biomedicine, HEALTH, Aarhus University, DK-8000 Aarhus C, Denmark \\ ${ }^{2}$ Department of Molecular Biology and Genetics, Science and Technology, Aarhus University, DK-8000 Aarhus C, Denmark \\ ${ }^{3}$ Department of Pediatrics, Stanford University, Stanford, California 94305, USA
}

\begin{abstract}
Our genes are post-transcriptionally regulated by microRNAs (miRNAs) inducing translational suppression and degradation of targeted mRNAs. Strategies to inhibit miRNAs in a spatiotemporal manner in a desired cell type or tissue, or at a desired developmental stage, can be crucial for understanding miRNA function and for pushing forward miRNA suppression as a feasible rationale for genetic treatment of disease. For such purposes, RNA polymerase II (RNA Pol II)-transcribed tough decoy (TuD) miRNA inhibitors are particularly attractive. Here, we demonstrate augmented miRNA suppression capacity of TuD RNA hairpins linked to the Woodchuck hepatitis virus post-transcriptional regulatory element (WPRE). This effect is positiondependent and evident only when the WPRE is positioned upstream of the TuD. In accordance, inclusion of the WPRE does not change nuclear export, translation, total levels of TuD-containing RNA transcripts, or cytoplasmic P-body localization, suggesting that previously reported WPRE functions are negligible for improved TuD function. Notably, deletion analysis of TuD-fused WPRE unveils truncated WPRE variants resulting in optimized miRNA suppression. Together, our findings add to the guidelines for production of WPRE-supported anti-miRNA TuDs.
\end{abstract}

Keywords: miRNA suppression; TuD; miRNA sponge; decoy; WPRE

\section{INTRODUCTION}

MicroRNAs (miRNAs), a class of noncoding RNAs, are crucial post-transcriptional regulators of gene expression with key roles in a wealth of cellular pathways and biological processes. MicroRNAs are transcribed from the genome as single primary miRNAs (pri-miRNAs) or clusters of pri-miRNAs, which are processed in several consecutive steps to become single-stranded miRNA molecules with a length of 20-24 nucleotides (nt). Post-transcriptional gene regulation mediated by mature miRNAs is accomplished by the RNA-induced silencing complex (RISC) recruited to mRNA typically through imperfect base-pairing between the miRNA and a recognition site located in the $3^{\prime}$ untranslated region (UTR) of the mRNA. Depending on the degree of complementarity between the miRNA and its target mRNA, miRNA-mediated regulation of gene expression is achieved either by direct endonucleolytic cleavage of the mRNA or by translational repression and activation of deadenylation and exonucleolytic decay (Winter et al. 2009; Fabian et al. 2010). The equilibrium between translation and repression/activation of decay of miRNA target mRNAs is believed to rely on the intricate tun-

Corresponding author: giehm@biomed.au.dk

Article is online at http://www.rnajournal.org/cgi/doi/10.1261/rna.061192. 117. ing between available decay enzymes and miRNA-bound messenger ribonucleoproteins (mRNPs). MicroRNA substrates and proteins that facilitate their repression and decay accumulate in cytoplasmic processing bodies (P-bodies), which also harbor numerous decay enzymes, RNA binding proteins, and additional labile mRNAs (for review, see Franks and Lykke-Andersen 2008; Decker and Parker 2012). At present, thousands of miRNAs and their cognate targets have been identified, and an increasing number of studies report correlations between disturbed miRNA expression and a variety of pathogenic conditions. This has triggered the need of effective tools that can regulate miRNA activity in basic cellular and animal models as well as demonstrate the potential of miRNAs as therapeutic targets for treatment of disease (McDermott et al. 2011).

The activity of a specific miRNA species or a family of related miRNAs can be managed by designed inhibitors that bind miRNAs with high affinity (Bak and Mikkelsen 2014). Such inhibitors may typically act through direct base-pairing

(C) 2017 Hollensen et al. This article is distributed exclusively by the RNA Society for the first 12 months after the full-issue publication date (see http://rnajournal.cshlp.org/site/misc/terms.xhtml). After 12 months, it is available under a Creative Commons License (Attribution-NonCommercial 4.0 International), as described at http://creativecommons.org/licenses/ by-nc/4.0/. 
to the mature miRNA and include synthetic oligonucleotides and locked nucleic acids (LNA) as well as small RNA inhibitors expressed from viral or nonviral vectors. Although synthetic miRNA inhibitors have been shown to suppress the function of specific miRNA in vitro as well as in vivo (Krützfeldt et al. 2005), the delivery of synthetic miRNA inhibitors is challenging in cell types and tissues that are difficult to transfect and access. Viral vectors, in contrast, allow efficient transfer of the miRNA inhibitors to many cell types and tissues and will within a population of cells allow for a more even distribution of inhibitors than can be achieved by conventional transfection methodologies. Furthermore, persistent expression of miRNA inhibitors is possible by the use of both viral and nonviral vector systems that allow genomic insertion of inhibitor-encoding gene cassettes in cells of interest. Also, the expression of vector-encoded miRNA inhibitors can be spatiotemporally regulated by the use of tissue-specific and inducible promoters, and the inhibitor can be produced in its most simple form as a short RNA molecule or fused to a protein-coding region encoding, for example, a fluorescent reporter protein, such as GFP (Bak et al. 2013a).

Like the majority of synthetic miRNA inhibitors, the most simple vector-encoded miRNA inhibitors are short RNAs fully complementary to the target miRNA (Scherr et al. 2007). However, several other designs with a varying number of successive miRNA binding sites and secondary structures have successfully been exploited for inhibition of miRNAs in vitro or in vivo (Gentner et al. 2009; Haraguchi et al. 2009; Ebert and Sharp 2010). Previously, we showed strong suppression of miRNA activity by RNA polymerase III (RNA Pol III)-transcribed tough decoy (TuD) miRNA inhibitors and found among a set of inhibitors expressed from both plasmids and lentiviral vectors that this type of inhibitor was particularly potent (Bak et al. 2013b). In addition, this study showed that the miRNA suppression activity was retained by RNA molecules in which the TuD sequence was fused to the $3^{\prime}$-end of an eGFP reporter gene and expressed from an RNA Pol II-driven promoter (Bak et al. 2013b). Different TuD delivery methods include standard gene transfer vehicles like lentiviral, adenoviral, and adeno-associated virus (AAV)-derived vectors, which have been found to facilitate transfer of TuDs to a wide range of cell types (Haraguchi et al. 2009; Lu et al. 2011; Sakurai et al. 2012; Xie et al. 2012; Mockenhaupt et al. 2015).

The TuD structure typically consists of a 122-nt-long hairpin-shaped structure with a larger internal, unpaired region containing two miRNA binding sites, one on each strand of the hairpin (Haraguchi et al. 2009). This region is flanked by longer stems, which serve to stabilize the structure and facilitate transport of the RNA Pol III-transcribed TuDs to the cytoplasm. Hence, the hairpin-shaped secondary structure was optimized by Haraguchi et al. (2009) for nuclear export by the Exportin-5 pathway, but also for prevention of Ago2mediated cleavage by insertion of a 4 -nt-long bulge sequence in the miRNA binding sites at the position opposite to nucleotides 10-11 of the bound miRNA. Another study showed more efficient miRNA suppression by synthetic TuDs made of 2'-O-methylated (2'-OMe) RNA than by standard synthetic miRNA inhibitor molecules (Haraguchi et al. 2012). The fate of miRNAs that are specifically targeted by TuDs through base-pairing is still debated (Haraguchi et al. 2009; Sakurai et al. 2012; Xie et al. 2012; Bak et al. 2013b). However, a high-throughput sequencing study has shown degradation of TuD-targeted miRNAs by the tailing and trimming pathway (Xie et al. 2012).

To expand the use of TuDs, we have previously refined the design of TuD-encoding vectors. First, we showed enhanced suppression of miRNA activity by RNA Pol II-transcribed clusters containing up to four consecutive TuDs, and thus up to a total of eight miRNA recognition sites (Bak et al. 2013b; Hollensen et al. 2013). Furthermore, we developed dual-targeting and multitargeting clusters of TuDs able to suppress the function of up to six unrelated miRNAs with nearly identical efficiency as standard TuD singletons (Hollensen et al. 2013). Standard and more advanced TuD designs can typically be used for identification of novel miRNA target genes and explored for treatment capacities ( $\mathrm{Lu}$ et al. 2011; Sakurai et al. 2012; Xie et al. 2012). In an elegant novel approach, Mockenhaupt et al. (2015) and Michler et al. (2016) recently demonstrated suppression of the sense strand of processed small hairpin RNAs (shRNAs) by DNA-encoded TuDs, leading to the alleviation of off-target activities facilitated by sense strand activity. Also, TuDs can be multiplexed in libraries for high-throughput screening of miRNAs involved in certain cellular pathways (Qiu et al. 2015).

The post-transcriptional regulatory element (PRE) originating from the mammalian hepadnavirus Woodchuck hepatitis virus (WHV) is widely used as a cis-acting gene regulatory element in viral vectors. The WHV PRE (WPRE) is a fixture in standard lentiviral vectors, but has been used also in adenoviral vectors, AAV-derived vectors, and nonviral vectors (Loeb et al. 1999; Zufferey et al. 1999; Salmon et al. 2000; Xu et al. 2003). Inserted in the sense orientation in the $3^{\prime}$-end of a transgene, the 590-nt-long WPRE RNA element enhances gene expression both in vitro and in vivo (Zufferey et al. 1999; Xu et al. 2003; Higashimoto et al. 2007). Due to its origin, WPRE contains remnants of the WHV genome, including a sequence encoding the first 60 amino acids (aa) of the 150-aa large X-protein and the promoter sequence driving expression of the X-protein. Previous studies have suggested a potential oncogenic effect of the X-protein and even of C-terminally truncated variants of the X-protein (Höhne et al. 1990; Wei et al. 1995; Kingsman et al. 2005). Hence, a potential oncogenic function of WPRE may be of concern in relation to the use of WPREcontaining vectors for gene delivery and therapeutic applications. However, the promoter sequence and start codon of the X-protein can be mutated and abrogated without influencing the cis-acting gene regulatory function of the WPRE 
(Schambach et al. 2006; Zanta-Boussif et al. 2009). Based on sequence similarities between WPRE and the PRE from the hepatitis $B$ virus (HBV), WPRE is assumed to increase nuclear RNA export as documented for the corresponding HBV element (Donello et al. 1996; Zufferey et al. 1999). Furthermore, the importance of the CRM1-dependent export pathway for WPRE function supports the notion that export of RNA is stimulated by the presence of the WPRE sequence (Popa et al. 2002). In accordance, WPRE increases nuclear export of otherwise retained transcripts encoded by the mutated myotonic dystrophy protein kinase (DMPK) gene causing myotonic dystrophy (Mastroyiannopoulos et al. 2005). Nevertheless, other studies have suggested that WPRE induces increased levels of both nuclear and cytoplasmic RNA (Donello et al. 1998; Zufferey et al. 1999), longer RNA half-life (Zufferey et al. 1999), and reduced readthrough transcription (Higashimoto et al. 2007; Schambach et al. 2007), all of which may promote increased viral titers in the context of retroviral vectors (Zufferey et al. 1999; Higashimoto et al. 2007; Schambach et al. 2007). In agreement with these diverging functions, the regulatory function of WPRE seems to be context-dependent (Schambach et al. 2000; Klein et al. 2006), and the exact underlying mechanism for WPRE-mediated enhanced transgene expression still remains to be deduced.

For purposes that demand effective miRNA suppression, guidelines are needed to design optimized DNA-encoded TuD miRNA inhibitors. Here, we demonstrate that the miRNA suppression capacity of RNA Pol II-transcribed TuD-containing RNAs is markedly augmented by the inclusion of WPRE in the transcript and that this effect is achieved only when the WPRE is located upstream of the TuD hairpin. Notably, a WPRE deletion analysis shows beneficial effects of WPRE deletion variants, resulting in the highest level of miRNA suppression using TuDs flanked by a WPRE devoid of the 206-nt-long $\alpha$-fragment corresponding to the middle one-third of the WPRE sequence. These data suggest that known WPRE functions are not relevant for improved TuD function and correlate with our findings that inclusion of the WPRE neither changes nuclear export, translation, total levels of TuD-containing RNA transcripts nor cytoplasmic P-body localization. Our findings suggest a new set of guidelines for production of RNA Pol II-transcribed TuDs.

\section{RESULTS}

\section{Enhanced microRNA suppression activity of RNA Pol II- transcribed TuD hairpins flanked on the upstream side by WPRE}

To study the influence of WPRE on suppression of miRNA activity by RNA Pol II-transcribed TuDs transiently expressed from plasmids, we generated vectors containing eGFP-fused TuDs targeting four unrelated miRNAs with or without an intervening WPRE (WPRE-TuD and TuD, respectively) (Fig.
1A). In addition, to control for the potential effect of the increased distance between the TuD and the eGFP reporter gene, we constructed control vectors with an antisense version of the WPRE placed between eGFP and the TuD (WPRErev$\mathrm{TuD}$ ) (Fig. 1A). All transcripts containing TuDs targeting miR-7, $-16,-21$, and -143 , respectively, were expressed from a CMV RNA Pol II promoter. The capacity of the TuD-containing transcripts to suppress miRNAs was evaluated in a dual-luciferase reporter assay in HEK-293 cells using a reporter plasmid containing either no or a perfect miRNA target site (miR-7, -16, -21, or -143) fused to a Renilla luciferase (RLuc) reporter gene. Expression of Firefly luciferase (FLuc) from another expression cassette in the plasmid allowed normalization for potential transfection variation. The normalized RLuc/FLuc ratio, determined $2 \mathrm{~d}$ after cotransfection with the TuD-containing vectors and the dual-luciferase reporter plasmid, was used as a measure of the capacity of the respective TuDs to suppress miRNA function. Due to relative low endogenous expression of two of the miRNAs (miR-7 and -143) in HEK-293 cells, a plasmid encoding a miRNA expression cassette was included in assays evaluating TuDs targeting these miRNAs. Overexpression of miR-7 and -143 after plasmid transfection was verified by RTq-PCR (Supplemental Fig. S1). For all four miRNAs, the RLuc/FLuc ratio was significantly higher in cells treated with WPRE-fused TuDs relative to cells expressing TuDs without the flanking WPRE (Fig. 1B), although some suppression of miRNA activity was indeed evident for transcripts (targeting miR-7, -16, and -21) containing the TuD alone. For two of the four targeted miRNAs, the WPRErev-TuD configuration was not capable of suppressing miRNA activity, whereas the activity was comparable to that of the TuD configuration for the two remaining miRNAs (Fig. 1B). Altogether, these findings confirm our previous observation that miRNAs are effectively suppressed by RNA Pol II transcripts harboring a WPRE-flanked TuD hairpin (Bak et al. 2013b). Moreover, the miRNA suppression potential of WPRE-fused TuDs is superior to both WPRE-devoid $\mathrm{TuD}$ motifs and TuDs flanked by the reverse oriented WPRE element.

To study whether the position of WPRE relative to the TuD hairpin has importance for efficacy of TuD-mediated miRNA suppression, we constructed eGFP-TuD-WPRE expression vectors encoding RNA transcripts with the WPRE located downstream from the $\mathrm{TuD}$ sequence (Fig. 1A). However, miRNA suppression assays consistently showed higher levels of suppression by TuDs that were flanked upstream by the WPRE (Fig. 1C). Hence, for all three studied miRNAs (miR-7, miR-16, and -21), TuDs fused to the $3^{\prime}$ end of WPRE were more potent inhibitors, whereas beneficial effects of WPRE in the downstream position were not evident. Based on these findings, we conclude that the WPRE needs to be situated upstream of the TuD to support optimized miRNA suppression.

To further validate the impact of WPRE on the miRNA suppression potential of the TuDs, we constructed lentiviral 
A
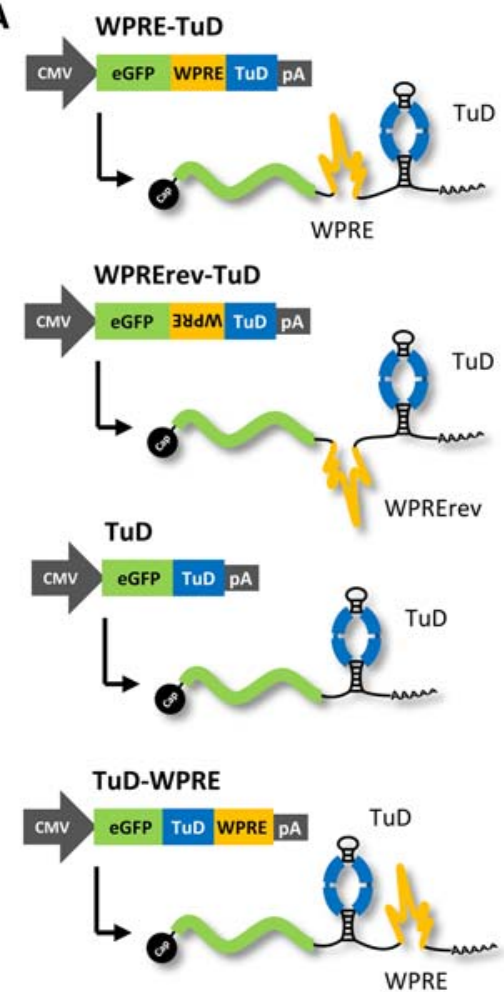

C

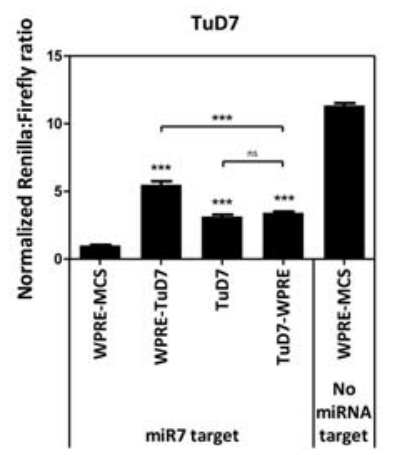

B
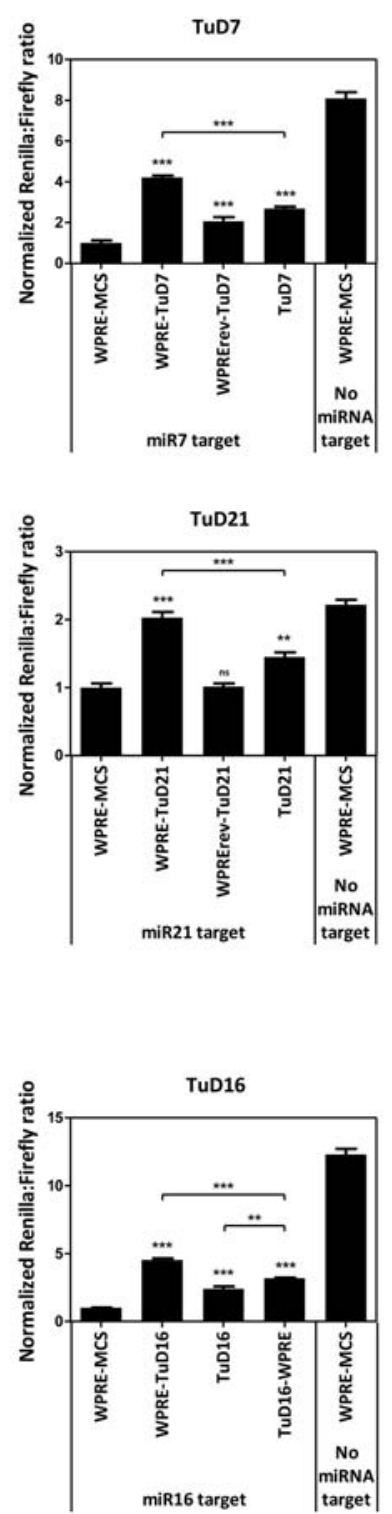
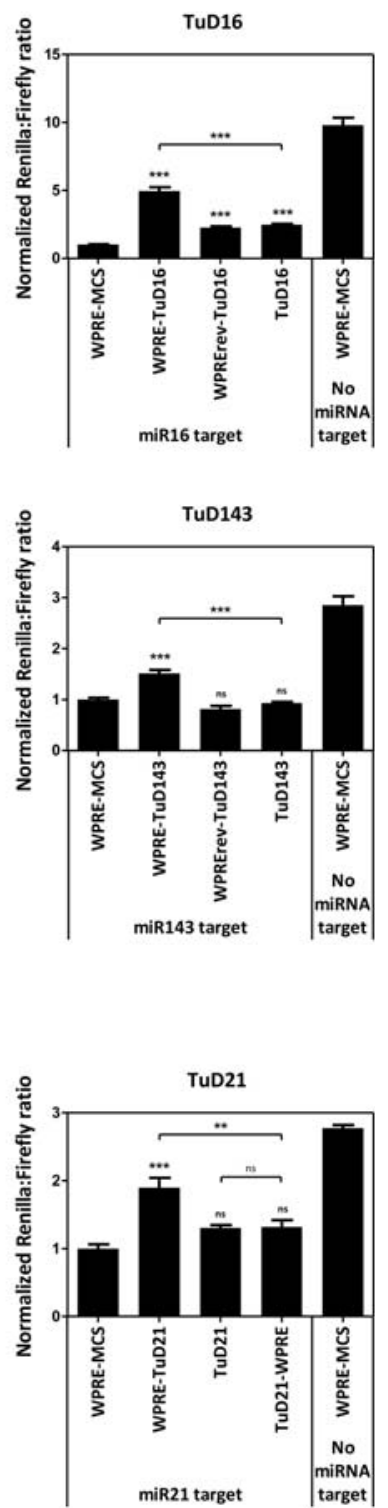

FIGURE 1. Fusion of WPRE to TuDs facilitates enhanced miRNA suppression after transient plasmid transfections. (A) Schematic representation of TuDs expressed from the CMV RNA Pol II promoter and fused to eGFP and either the $3^{\prime}$-end (WPRE-TuD) or $5^{\prime}$-end (TuD-WPRE) of WPRE, eGFP and the $3^{\prime}$-end of antisense WPRE (WPRErev-TuD), or directly to eGFP (TuD). A schematic representation of the TuD hairpin is provided in Hollensen et al. (2013). (B) Levels of miR-7, -16, -21, and -203 suppression were measured by dual-luciferase assays using a reporter plasmid encoding an RLuc reporter gene made responsive to miRNA binding by a perfect miRNA target site. $(C)$ Dual-luciferase assay comparing miRNA suppression by WPRE-TuD and TuD-WPRE. Data are depicted as mean + SEM. Asterisks above columns show $P$-values for comparison with the negative control WPRE-MCS cotransfected with psiCHECK-vector containing a perfect miRNA target site. Asterisks indicate significance as follows: $\left({ }^{* *}\right) P<0.01$, $(* * *) P<0.001$, (ns) not significant.

plasmids containing eGFP-fused TuDs with and without intervening WPRE (WPRE-TuD and TuD, respectively) (Fig. 2A). The TuD-containing transcripts were expressed from the PGK RNA Pol II promoter and designed to target either of three unrelated miRNAs (miR-16, -21, and -203). In the case of miR-203, which is poorly expressed in HEK-293 cells, we also transfected a miR-203 expression plasmid together with the RLuc/FLuc reporter (Supplemental Fig. S1). In accordance with the data shown in Figure 1B, dual-luciferase assays after transient transfections of HEK-293 cells with the lentiviral plasmids showed significantly higher levels of miRNA suppression with WPRE-TuDs than with TuDs that were not flanked by WPRE (Supplemental Fig. S2). After lentiviral production using the TuD-encoding transfer plasmids, transductional titers were determined based on flow cytometry analysis of eGFP expression in transduced $\mathrm{HeLa}$ cells. For all TuD-containing vectors, WPRE gave rise to a titer increase (Fig. 2B). Although not significant, a similar 
A
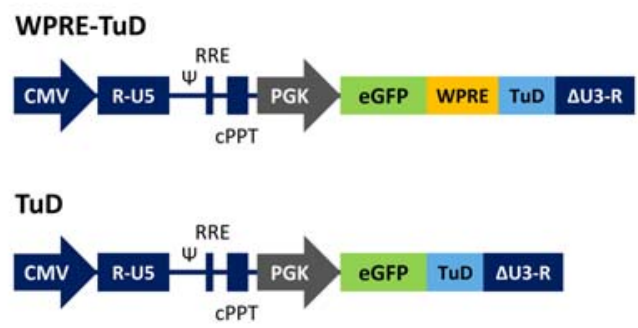

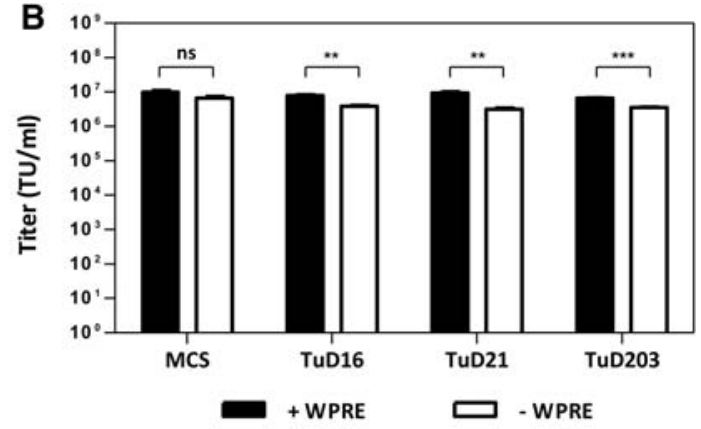

TuD21
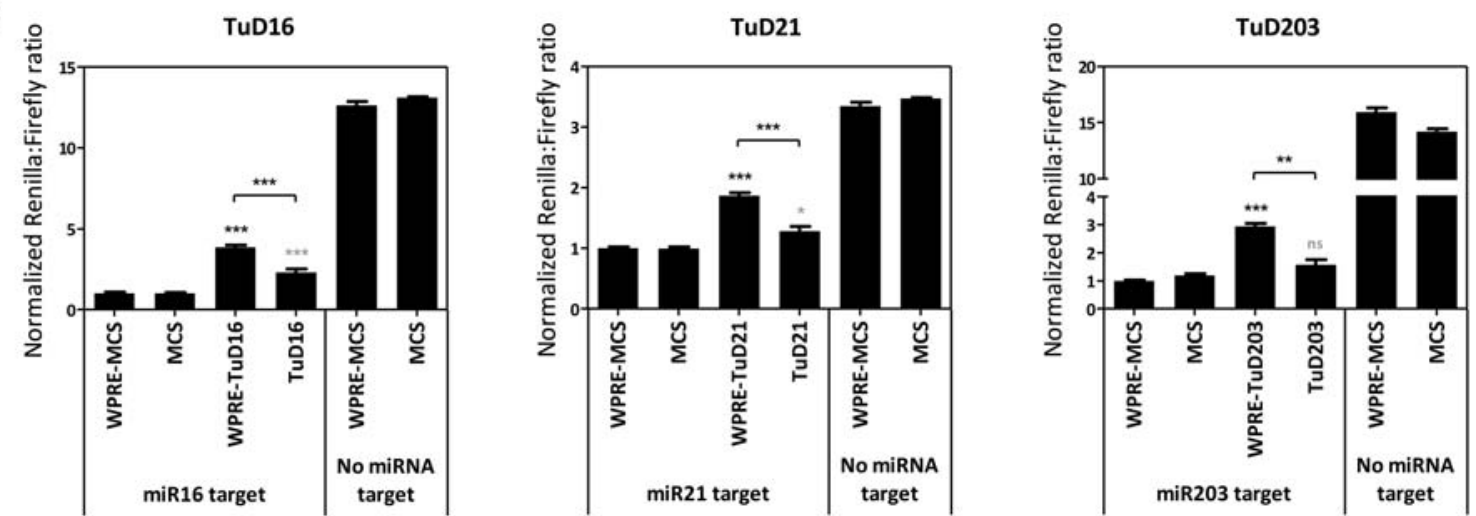

FIGURE 2. WPRE in lentiviral vectors increases titers and miRNA suppression activity of TuDs. $(A)$ Schematic representation of the lentiviral vector plasmid encoding eGFP- and WPRE-fused TuDs expressed from the PGK RNA Pol II promoter. (B) Transductional titers of lentiviral vectors encoding eGFP-fused TuDs with and without intervening WPRE determined by flow cytometric analysis of eGFP expression. $(C)$ Dual-luciferase assay showing improved miRNA suppression activity of WPRE-fused TuDs targeting miR-16, -21, and -203 after lentiviral transduction using an MOI of 100 for all TuD-encoding lentiviral vectors. Included controls are similar for experiments performed with TuD16 and TuD21. Data are depicted as mean + SEM. Asterisks above columns show $P$-values for comparison with the negative control WPRE-MCS (asterisks in black) or MCS (asterisks in gray) cotransfected with psiCHECK-vector containing a perfect miRNA target site. TU, transducing units; $\left(^{*}\right) P<0.05,\left({ }^{* *}\right) P<0.01,\left({ }^{* * *}\right) P<0.001$, (ns) not significant.

tendency was seen also for the control vectors without any TuDs (Fig. 2B). These findings reproduced the observations that originally led to the inclusion of WPRE in standard lentiviral vectors (Zufferey et al. 1999).

Based on the measured titers, dual-luciferase assays were carried out in order to evaluate the miRNA suppression activity of WPRE-fused TuDs after lentiviral transduction of HEK-293 cells. One day after transduction, using an estimated multiplicity of infection (MOI) of 100 of TuD-encoding lentiviral vectors, the RLuc/FLuc reporter plasmid was delivered to the cells by plasmid transfection. Luciferase activities were measured $2 \mathrm{~d}$ after transfection. Corroborating previous findings, transduction using lentiviral vectors encoding WPRE-fused TuDs resulted in robust miRNA suppression (Bak et al. 2013b), whereas TuDs without the flanking WPRE remained significantly weaker suppressors even when the virus load was normalized for the differences in MOI (Fig. 2C). Hence, consistent with the data obtained with TuDs expressed from transfected plasmids, the function of lentivirally delivered TuDs, expressed by an RNA Pol II promoter, was positively affected by the WPRE positioned upstream of the TuD hairpin.

\section{Neither altered RNA levels nor changes in nuclear RNA export explain WPRE-dependent TuD function}

Our discovery of the position-dependent impact of WPRE on $\mathrm{TuD}$ function supported the notion that the WPRE sequence itself, rather than unique functional properties of the WPRE, assisted TuD activity. Nevertheless, to investigate the impact further, we embarked upon a series of basic analyses focusing on processing of WPRE-containing transcripts. Since the WPRE is typically assumed to affect transgene expression at a post-transcriptional level (Zufferey et al. 1999; Popa et al. 2002; Higashimoto et al. 2007), we examined whether the WPRE caused changes in overall RNA levels, nuclear RNA export, and translation efficiency of TuD-containing RNA Pol II transcripts produced from a CMV promoter. Since TuD-mediated miRNA suppression is believed to predominantly occur in the cytoplasm, an altered rate of nuclear RNA export could have a pronounced influence on the miRNA suppression potential of the TuD miRNA inhibitors. To study the effect of WPRE on both RNA levels and nuclear RNA export, we initially performed RT-qPCR with eGFPspecific TaqMan primers and probes. Total and cytoplasmic 
RNA was harvested $2 \mathrm{~d}$ after transfection of HeLa cells with WPRE-fused TuDs targeting miR-7 and -16. As shown in Supplemental Figure S3B, we found by this method that WPRE caused a reduction in total and cytoplasmic eGFP RNA levels for vectors expressing the MCS and TuD7 transcripts. Most importantly, the ratio between cytoplasmic and total eGFP RNA levels of TuD-encoding RNA was not affected by the WPRE, indicating that nuclear export was unaffected (Fig. 3A). Purity and quality of the cytoplasmic cell fractions were verified by RT-qPCR specific for the nuclear localized small nucleolar RNA U48, which showed only negligible levels of $U 48$ in the cytoplasmic fraction compared to both the nuclear and total RNA fractions (Supplemental Fig. S3A).

To further support the observations based on RT-qPCR analysis, RNA fluorescence in situ hybridization (FISH) was performed using eGFP-specific probes $2 \mathrm{~d}$ after transfection of HeLa cells with vectors encoding eGFP-fused, miR16targeting TuDs with and without intervening WPRE. Independent of the presence of WPRE or TuDs, the eGFPencoding RNA was primarily localized in the cytoplasm (on average $75 \%$ of the RNA) (representative example shown in Fig. 3B). Furthermore, we reproducibly found that the levels of total, cytoplasmic, and nuclear eGFP RNA, as determined by quantification of FISH signals within more than 50 cells, were reduced after inclusion of WPRE in vectors both with and without TuDs (Fig. 3C). As a measure of the rate of nuclear export of the WPRE- and TuD-encoding RNA, ratios between cytoplasmic and nuclear eGFP RNA were calculated and plotted for all analyzed cells (Fig. 3D). For both RNAs with and without TuDs targeting miR-16, this analysis demonstrated that WPRE did not affect the steady-state ratio of nuclear/cytoplasmic eGFP RNA transcripts, suggesting that the nuclear export rates were not significantly changed (Fig. 3D). To rule out that a potential positive effect of WPRE on nuclear eGFP RNA export was hidden by too high RNA levels in the cells, RNA-FISH was carried out using HeLa cells transfected with lower amounts of plasmid DNA. Although we observed in this case only a tendency to a reduction in total, cytoplasmic, and nuclear eGFP RNA levels after inclusion of WPRE (Supplemental Fig. S4A), we again found that the rate of nuclear export of TuD-encoding RNA was not affected by the WPRE (Supplemental Fig. S4B). In summary, our findings show that WPRE does not positively support nuclear export of RNA Pol II eGFP transcripts. We conclude that the enhanced miRNA suppression activity of WPRE-flanked $\mathrm{TuD}$ hairpins is not facilitated by increased nuclear export of TuD-containing transcripts.

\section{Transgene expression is not increased by the presence of WPRE}

To assess the influence of WPRE on translation and transgene protein expression, flow cytometry analysis of eGFP expression was carried out $2 \mathrm{~d}$ after transfection of HeLa cells with
A
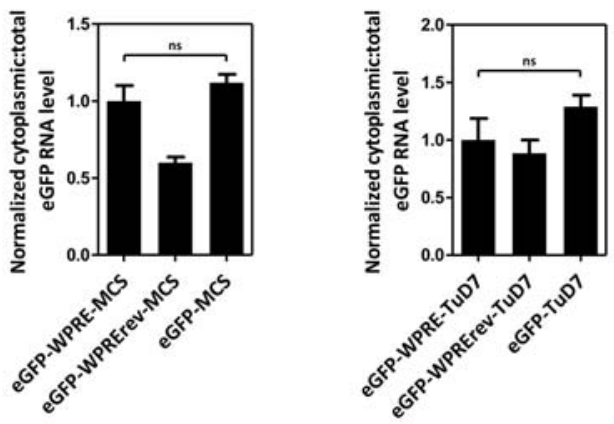

C
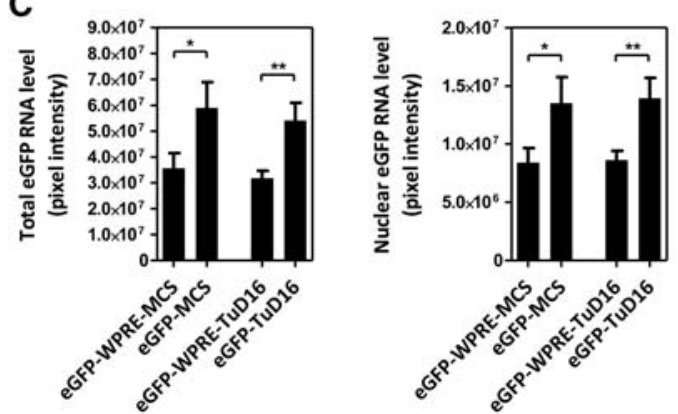

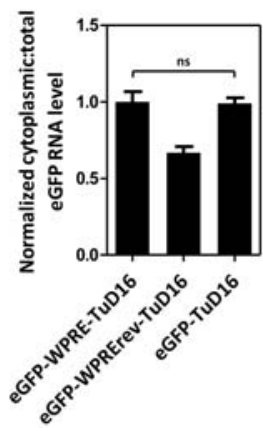

B
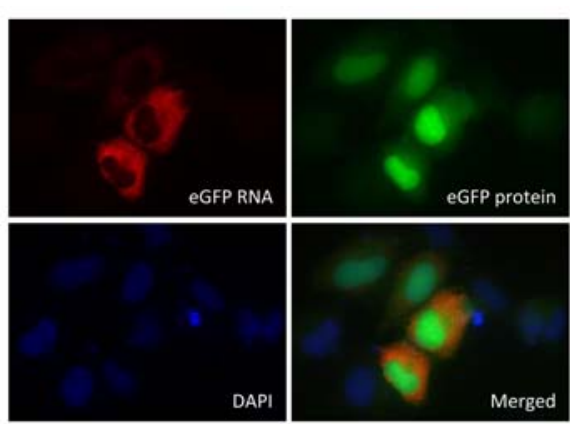

D
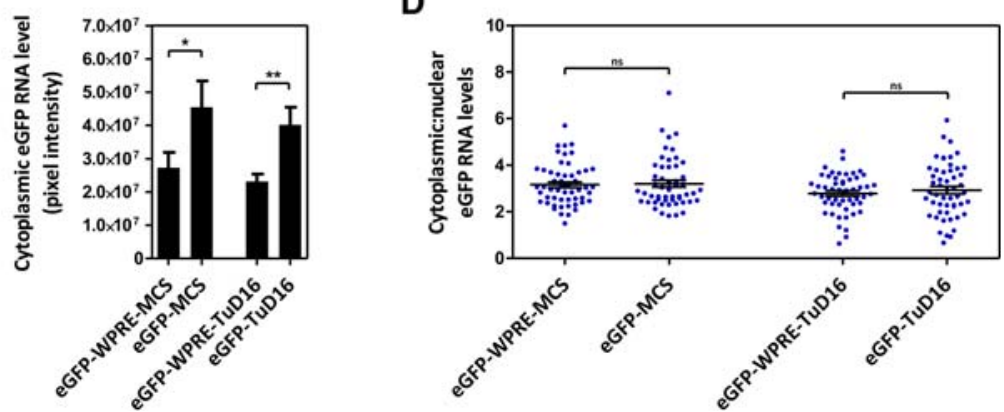

FIGURE 3. Unchanged rates of nuclear RNA export by fusion of TuDs to WPRE. The nuclear export rate of TuD-encoding RNA evaluated as the ratio between cytoplasmic and total eGFP RNA levels after eGFP-specific RT-qPCR $(A)$ or as the ratio between cytoplasmic and nuclear eGFP RNA levels after RNA-FISH analysis $(D)$. (B) Example of RNA-FISH staining using eGFP-specific probes in HeLa cells transfected with 400 ng of plasmid containing WPRE-TuD16, TuD16, WPRE-MCS, or MCS. (C) Total, nuclear, and cytoplasmic eGFP RNA levels measured as pixel intensities after FISH staining. Data are depicted as mean + SEM. $\left(^{*}\right) P<0.05,\left({ }^{* *}\right) P<0.01$, (ns) not significant. 
plasmids encoding WPRE-TuDs, WPRErev-TuDs, or TuDs targeting miR-7 and -16 (Supplemental Fig. S5A). For MCS constructs that did not contain any $\mathrm{TuD}$, we observed that median fluorescence intensity (MFI) was highest in the absence of the WPRE sequence (Supplemental Fig. S5B, lower panel). The same tendency, although not statistically significant, was evident also for the TuD-encoding vectors (Supplemental Fig. $\mathrm{S} 5 \mathrm{~B}$, lower panel). However, the percentage of eGFP-positive cells was comparable for all WPRE-TuD, WPRE-MCS, TuD, and MCS vectors (39\% to 54\% eGFP-positive cells) (Supplemental Fig. S5A, upper panel). To directly measure the translational initiation efficiency of the various transcripts and assess a potential impact of the WPRE on translation, polysome profiling was carried out for the WPRE-MCS, WPRErev-MCS, and MCS vectors (Supplemental Fig. S5C). The main fraction of the polysome-associated eGFP-encoding RNA produced from the WPRE-MCS and MCS constructs was found in fraction 6-12 (Supplemental Fig. S5C, 90\% in fraction 6-12), indicating a high translation efficiency of both these transcripts irrespective of WPRE inclusion. The WPRErev-MCS control displayed inefficient polysome-loading consistent with poor translation initiation efficiency and low steady-state GFP levels (Supplemental Fig. S5C). Together, these findings indicate that the presence of WPRE does not lead to increased eGFP expression.

\section{Localization of TuD-containing RNA transcripts to P-bodies is facilitated by the TuD, but occurs independently of the WPRE}

P-bodies are cytoplasmic sites of mRNA repression and decay in which labile mRNAs, such as miRNA targets, are known to accumulate (Franks and Lykke-Andersen 2008; Decker and Parker 2012). We therefore wanted to investigate the subcellular localization of RNA Pol II-transcribed TuDs and unveil any potential influence of WPRE on their potential recruitment to P-bodies. To this end, we performed eGFP-specific RNA-FISH combined with immunostaining for the P-body marker hDcpla in HeLa cells transfected with vectors encoding eGFP-fused TuD with and without WPRE. To avoid too high eGFP RNA levels, HeLa cells were subjected to fluorescence-activated cell sorting (FACS) based on eGFP expression levels $2 \mathrm{~d}$ after transfection. Cells with an intermediate eGFP expression level were selected for further analysis. As depicted in Figure 4A, distinct RNA foci that colocalized with hDcpla were seen after transfection with TuD-encoding vectors regardless of the presence of WPRE and WPRErev (Fig. 4A, left three panels). Importantly, since we did not observe any accumulation of the TuD-deficient eGFP RNA in P-bodies (Fig. 4A, right panels), the recruitment to P-bodies involves interactions between the TuD embedded in the transcript and likely the target miRNAs.

To study the importance of recruiting TuD-containing transcripts to P-bodies for the potential to suppress miRNA function, we performed siRNA-mediated knockdown of the P-bodies-associated proteins Lsm4 and DDX6. Initially, depletion of P-bodies after Lsm4 and DDX6 knockdown was verified by immunostaining using antibodies targeting the $\mathrm{P}$ body-specific markers Dcp1a and DDX6 (Supplemental Fig. S6A). Counting showed $58 \%$ and $88 \%$ reduction in the number of cells containing P-bodies after knockdown of Lsm 4 and DDX6, respectively, whereas P-bodies were found in $97 \%$ of the untransfected control cells and in $93 \%$ of the cells transfected with a scrambled siRNA sequence (Supplemental Fig. S6B). Under these conditions, dual-luciferase assays showed levels of miR-21 suppression in the presence of the WPRE that were approximately $1.3 \times$ higher than without WPRE (Fig. 4B). Notably however, these relative levels of suppression were similar in cells treated with all types of siRNAs as well as in untreated control cells (Fig. 4B), indicating that $\mathrm{TuD}$ and WPRE function was not affected by the depletion of P-bodies. In summary, TuD-containing miRNA-sponging transcripts are, as are normally miRNA-targeted mRNAs, recruited to P-bodies, by a mechanism that is independent of the WPRE. Also, miRNA suppression by TuDs occurs through mechanisms that do not involve recruitment to P-bodies.

\section{Enhanced TuD function is not driven by internal WPRE promoter sequence}

Due to the presence of the cryptic X-protein promoter (Schambach et al. 2006), the WPRE may have residual promoter activity that could potentially drive expression of short RNAs, like a short TuD-containing transcript. To test whether such promoter activity of WPRE has an impact on the enhanced miRNA suppression activity of WPRE-fused TuDs, we constructed vectors (WPRE $\Delta \mathrm{P}-\mathrm{TuD}$ ) encoding TuDs fused to a WPRE in which we introduced a 22-nt-long deletion including the X-protein promoter and part of the start codon for the X-protein located in the WPRE (nt 14821504; GenBank accession number J04514) (Fig. 5A). In addition, to restrict the production of full-length transcripts, vectors lacking the CMV promoter were produced. In the absence of the CMV promoter, constructs harboring WPRE-fused TuDs targeting miR-7 and - 16 did not show evidence of miRNA suppression (Fig. 5B). Furthermore, elimination of the X-protein promoter did not affect the efficiency of TuDs targeting miR-7 (Fig. 5B), and only a very small reduction in $\mathrm{TuD}$ function was observed for TuDs targeting miR-16 (Fig. 5B). In conclusion, the X-protein promoter is not capable of driving TuD expression and can be eliminated without affecting WPRE-mediated enhancement of miRNA suppression by TuDs.

\section{Enhanced miRNA suppression by TuDs flanked by truncated WPRE variants}

Together, our findings suggest that the impact of WPRE on $\mathrm{TuD}$ function is unlikely to reflect separate functional properties of WPRE affecting the miRNA suppression potential. This 

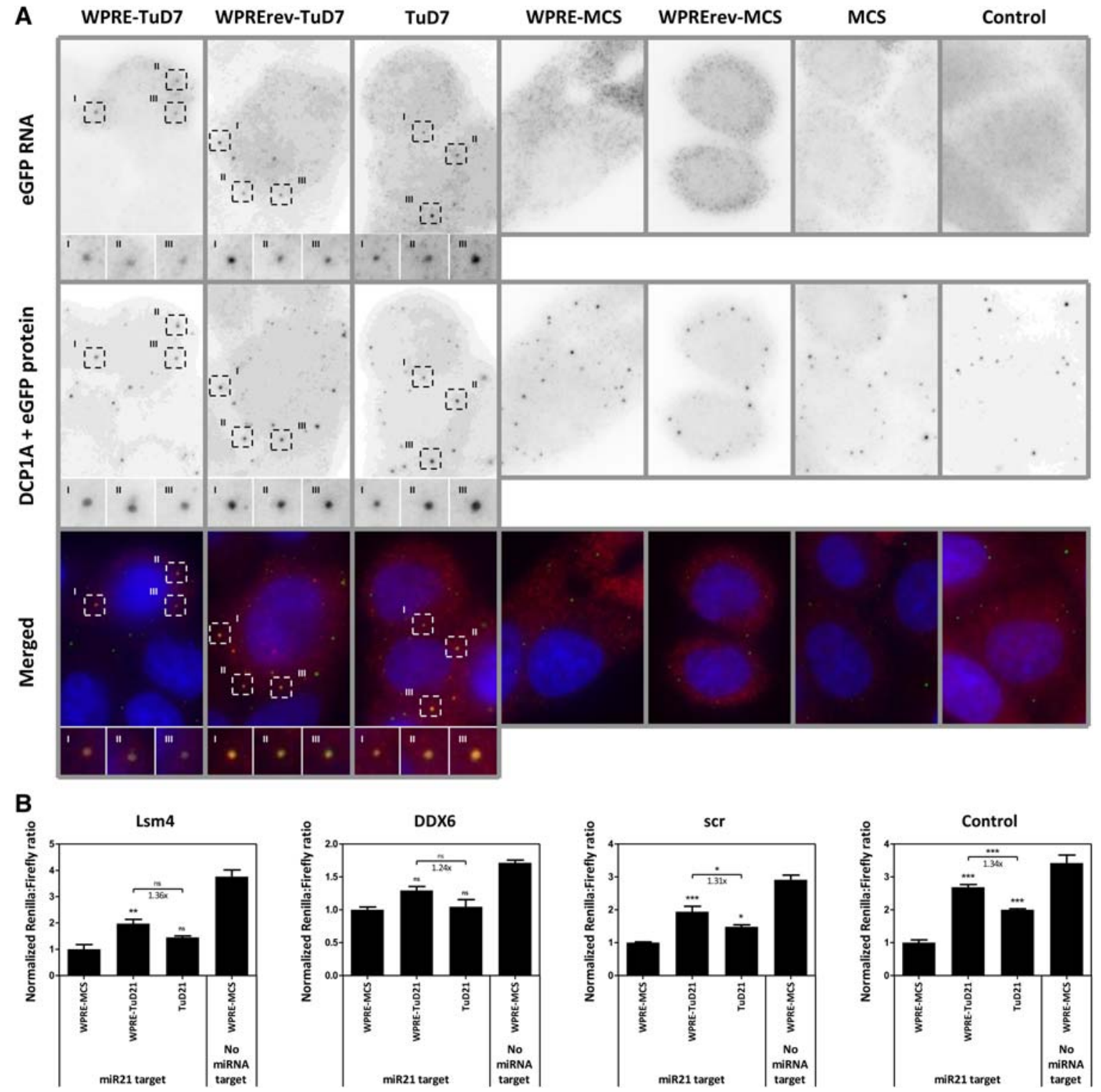

FIGURE 4. Colocalization between TuD-encoding RNA and P-bodies regardless of fusion of the TuDs to WPRE. (A) Combined RNA-FISH using eGFP-specific probes and immunostaining with antibodies targeting the P-body-specific marker hDcpla shows colocalization between TuD-encoding RNA and P-bodies for WPRE-TuD7, WPRErev-TuD7, and TuD7 whereas no colocalization was observed for WPRE-MCS, WPRErev-MCS, and MCS after transient transfections of HeLa cells. Due to Alexa488 labeling of the secondary antibodies, eGFP protein and hDcpla were visualized using the same filter. (B) Dual-luciferase assays evaluating levels of miR-21 suppression mediated by WPRE-TuD21 and TuD21 after knockdown of the P-body associated proteins Lsm4 and DDX6 using siRNA. Scrambled (scr) siRNAs and untransfected cells were used as controls. Data are depicted as mean + SEM. Asterisks above columns show $P$-values for comparison with the negative control WPRE-MCS cotransfected with psiCHECK-vector containing a perfect miRNA target site. Asterisks indicate significance as follows: $\left(^{*}\right) P<0.05,\left(^{* *}\right) P<0.01,\left(^{* * *}\right) P<0.001$, (ns) not significant.

led to the idea that the TuD hairpin benefits from the nearby sequence and that $\mathrm{TuD}$ function could be even further improved by altering the WPRE sequence. To test this hypothesis, we analyzed the miRNA suppression capacity of a set of constructs encoding TuDs fused to WPRE deletion variants (listed in Fig. 5A). Since WPRE contains a long hairpin-shaped secondary RNA structure (Donello et al. 1998), we first tested whether this hairpin was supporting potent miRNA suppression, potentially by supporting proper $\mathrm{TuD}$ folding. However, deletion of the hairpin (WPRE $\Delta$ ahairpin) did not have a major impact on miRNA suppression (compare suppression by eGFP-WPRE-TuD and eGFP-WPRE $\triangle$ ahairpin-TuD in Fig. 5C), showing that the hairpin was dispensable for improved $\mathrm{TuD}$ function.

To elucidate whether the impact of WPRE could even be improved by deleting specific WPRE domains, we tested the function of TuDs fused to six different WPRE variants harboring different deletions of $\gamma^{-}, \alpha$-, and $\beta$-subdomains 
A

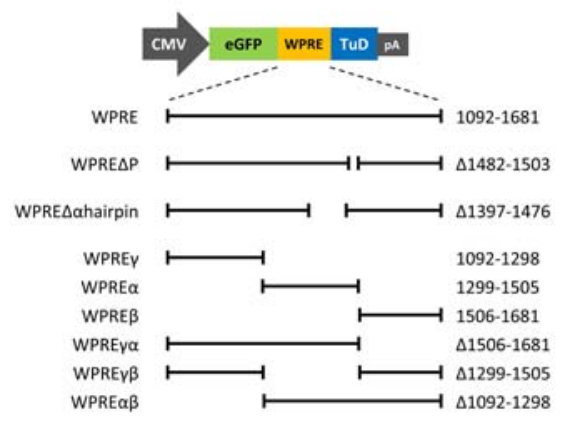

C

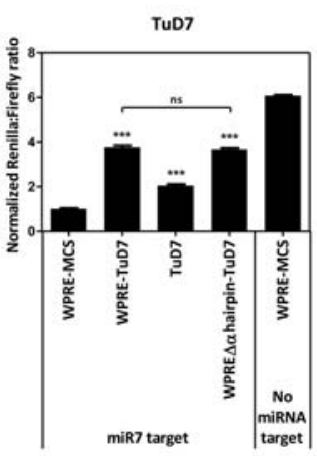

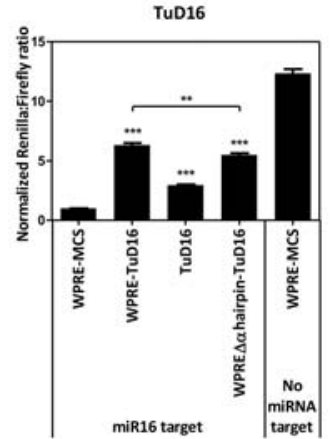

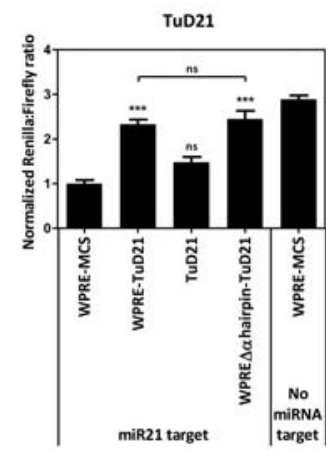

B

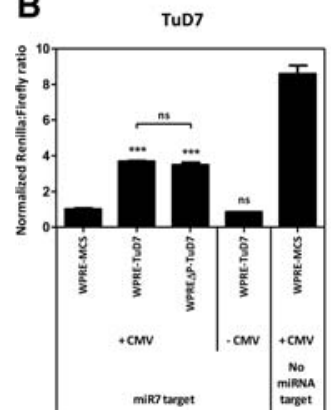

TuD16

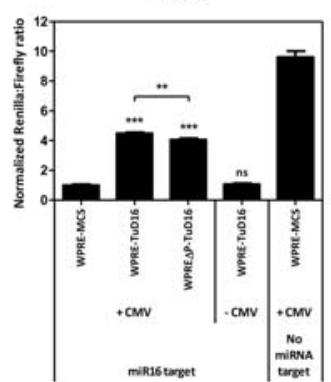

D

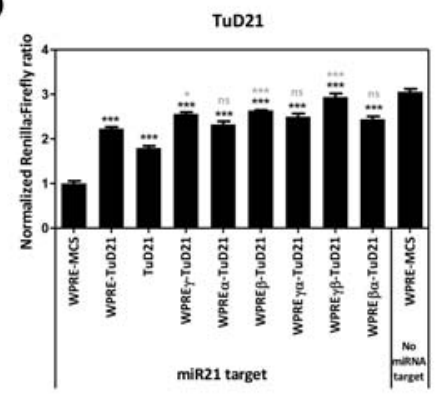

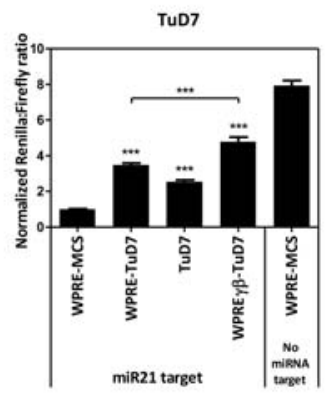

FIGURE 5. Truncation of WPRE further enhances TuD function. (A) Schematic representation of WPRE-fused TuDs with deletion in WPRE (numbers refer to nucleotides of WPRE present or deleted according to GenBank accession number J04514). Evaluation of the impact of the X-protein promoter in WPRE $(B)$, the hairpin-shaped part of the $\alpha$-subunit $(C)$, and the $\gamma_{-}^{-}, \alpha$-, and $\beta$-subunits of WPRE $(D)$ on miRNA suppression activity of TuDs. Data are depicted as mean + SEM. Black asterisks above columns show P-values for comparison with the negative control WPRE-MCS cotransfected with psiCHECK-vector containing a perfect miRNA target site; gray asterisks above columns show $P$-values for comparison with WPRE-TuD cotransfected with psiCHECK-vector containing a perfect miRNA target site. $\left(^{*}\right) P<0.05,\left({ }^{* *}\right) P<0.01,\left({ }^{* * *}\right) P<0.001,($ ns) not significant.

that were previously defined by Donello et al. (1998). Notably, for both studied miRNAs, miR-21 and miR-7, the a-subdomain of WPRE had a negative impact on the overall effect of the WPRE on TuD function (Fig. 5D). Hence, for TuDs targeting miR-21 and miR-7, respectively, a WPRE variant harboring the $\gamma$ - and $\beta$-subdomains only (WPRE $\gamma \beta$ ), supported the most potent suppression of miRNA function that we have observed to date. In summary, our data demonstrate that the miRNA suppression capacity by RNA Pol IItranscribed TuDs is enhanced by an upstream WPRE sequence and that anti-miRNA effects can be further maximized by specific modulations of the WPRE sequence.

\section{DISCUSSION}

MicroRNAs play roles in almost all cellular processes and are implicated in numerous pathogenic conditions. This encourages development of optimized anti-miRNA agents for use in vitro as well as in vivo. Synthetic miRNA inhibitors targeting miR-122 have been used with long-term safety and efficacy for genetic treatment of Hepatitis C virus infections in clinical trials (Janssen et al. 2013; van der Ree et al. 2014). Although the use of synthetic miRNA inhibitors is being implemented in the clinic, treatment of many diseases would re- quire repeated administration. Moreover, unspecific and inefficient delivery remains a significant challenge using synthetic miRNA inhibitors for treatment of disease as well as for studies of miRNA biology both in vitro and in vivo. For many applications, exploiting vector delivery for administration of DNA-encoded miRNA inhibitors represents an attractive alternative that may confer long-term and tailored expression in most tissues and cell types. Optimized use of this tool obviously requires choices between vector types, promoters, and design of the miRNA-sponging transcript.

Previously, we compared seven different designs of miRNA inhibitors with varying numbers of miRNA binding sites and secondary structures and found potent miRNA suppression by RNA Pol III-transcribed TuDs after delivery by both plasmid transfections and lentiviral transduction (Bak et al. 2013b). We also showed that TuDs maintain their miRNA suppression potential after fusion to an RNA Pol II-transcribed gene, and for most TuDs the corresponding miRNAs were even more efficiently suppressed by TuDs transcribed from the PGK RNA Pol II promoter than from the H1 RNA Pol III promoter (Bak et al. 2013b). The robustness of RNA Pol II-transcribed TuDs allows expression of TuDs from almost any promoter, including spatiotemporally regulated promoters. In the present study, we demonstrated 
that the miRNA suppression potential of the TuD hairpin strongly depends on the presence of the cis-acting gene regulatory element WPRE situated upstream of the TuD. For all studied miRNAs, the WPRE facilitated a significant increase in the miRNA suppression activity of the TuDs after both plasmid transfection and lentiviral transduction. Notably, TuD function did not benefit from a WPRE sequence inserted downstream from the TuD hairpin. Consistent with previous findings for retroviral vectors, including lentiviral vectors (Schambach et al. 2006; Higashimoto et al. 2007), we obtained an increased viral titer after fusion of the TuDs to WPRE. Even after adjustment for the enhanced viral titer mediated by the WPRE, the WPRE-enhanced miRNA suppression activity of the TuDs was observed. Our findings support the notion that DNA-encoded TuDs should be fused to the $3^{\prime}$-end of the WPRE module for optimal potency. In all our vectors harboring WPRE in the upstream position, the WPRE was localized either 41 or $42 \mathrm{nt}$ upstream of the TuD hairpin; as of yet it remains unexplored whether the exact distance between the WPRE and TuD sequences is vital for the beneficial effect. However, supported by a wide range of studies that could not pinpoint the functional properties of WPRE that enhance TuD function (discussed below), we generated a panel of WPRE deletion variants and found improved $\mathrm{TuD}$-supporting functions in several of these variants. Constructs devoid of the WPRE a-subdomain were particularly effective and resulted in the highest level of miRNA suppression that we have seen with RNA Pol II-driven transcripts to date. This adds to a new set of guidelines for design of RNA Pol II-transcribed miRNA inhibitors. At this stage, however, it remains unclear as to why WPRE and in particular truncated versions support $\mathrm{TuD}$ function, but our controls (e.g., constructs harboring WPRE in the reverse orientation) suggest that the supportive effect is not the mere result of a certain beneficial distance, for example, between the eGFP stop codon and TuD hairpin.

Due to the presence of a cryptic promoter for the X-protein and the first 60 amino acids of the WHV X-protein, which has a potential oncogenic function, the use of WPRE in viral vectors has been debated (Kingsman et al. 2005). However, previous studies have shown that the promoter sequence and start codon of the X-protein can be abolished without influencing the cis-acting gene regulatory function of WPRE (Schambach et al. 2006; Zanta-Boussif et al. 2009). In one deletion variant, we examined the influence of the X-protein promoter on the enhanced miRNA suppression. Notably, the WPRE-embedded X-protein promoter was not able to drive the expression of the TuDs on its own, and elimination of the X-promoter overall did not disturb the beneficial impact on miRNA suppression activity. Thus, potential promoter activity of the WPRE was not responsible for the enhanced miRNA suppression activity mediated by WPRE.

WPRE is thought to affect the transcriptional and posttranscriptional level of transgene expression, and fusion of
WPRE to transgenes has been claimed to increase levels of both nuclear and cytoplasmic RNA and to enhance nuclear RNA export (Donello et al. 1998; Zufferey et al. 1999; Popa et al. 2002; Mastroyiannopoulos et al. 2005). Therefore, we carefully explored RNA levels, nuclear RNA export, transgene expression, and translational rates along with patterns of subcellular localization to unveil potential mechanisms of action for the observed increased miRNA suppression activity of the WPRE-fused TuDs. However, after plasmid delivery of WPRE-fused TuDs, we measured either unchanged or reduced RNA and protein levels and thus not an increased transgene expression mediated by WPRE. Consistent with results obtained by both Zufferey et al. (1999) and Higashimoto et al. (2007), we did not observe an increased nuclear RNA export rate of the WPRE- and TuD-encoding RNA that could explain the enhanced miRNA suppression activity of the TuDs after fusion with WPRE. Furthermore, we examined the subcellular localization of the RNA Pol II-transcribed TuDs. As previously shown by Haraguchi et al. (2009) for RNA Pol III-transcribed TuDs, we demonstrated that RNA Pol II-transcribed TuDs are localized mainly in the cytoplasm. Also, clear colocalization between TuDs and the P-body-specific marker hDcplA was observed regardless of the presence of WPRE. Importantly, however, eGFP-encoding RNA transcripts were recruited to P-bodies only when they contained a miRNA-targeting $\mathrm{TuD}$, supporting the notion that miRNAbound $\mathrm{TuD}$-containing transcripts are recruited to or even nucleate P-bodies for potential degradation. This is likely mediated by RISC-associated components such as Ago-proteins and GW182, which are known to accumulate in P-bodies along with their mRNA targets (Eystathioy et al. 2003; Ding et al. 2005; Sen et al. 2005; Parker and Sheth 2007; Franks and Lykke-Andersen 2008). However, TuD function and the supporting role of WPRE were evident even in P-bodydepleted cells, suggesting that miRNA suppression does not require recruitment to P-bodies.

Previous studies by Haraguchi et al. (2012) showed reduced miRNA suppression activity of synthetic TuDs after reduction of the length of the TuD stem, which may suggest that the miRNA suppression potential of the TuDs compared to other inhibitor designs benefit from a rigid and stable conformation of the TuDs. In addition, the fact that TuDs containing only two miRNA binding sites are capable of sponging miRNAs with an activity that is comparable to longer transcripts carrying eight successive miRNA recognition sites (Bak et al. 2013b) lends further support to the notion that a stable secondary structure of the TuDs is important for efficient miRNA suppression. We speculate that formation of this structure might be supported by the flanking WPRE sequence. In summary, we demonstrate that the inclusion of WPRE fused to the $5^{\prime}$-end of the TuD is desired for optimal activity of RNA Pol II-transcribed TuD miRNA inhibitors. Together, our studies provide new guidelines for design and production of optimized miRNA sponges. 


\section{MATERIALS AND METHODS}

All methods and associated references are available as Supplemental Material.

\section{Methods summary}

eGFP-fused TuDs with or without an intervening WPRE were expressed from CMV or PGK RNA Pol II promoters and delivered to cells by either expression plasmids or lentiviral vector. Third-generation lentiviral vectors were produced in HEK-293T cells, as previously described (Jakobsen et al. 2009). Vectors for expression of pri-miRNAs and psiCHECK vectors carrying perfect miRNA target sites in the $3^{\prime}$-UTR of the RLuc reporter gene were made as previously described in Primo et al. (2012), Bak et al. (2013b), and Hollensen et al. (2013). Dual-Glo Luciferase Assay System (Promega) was used to evaluate the miRNA suppression potential of the TuD miRNA inhibitors delivered by both plasmid transfections and lentiviral transductions. Lentiviral titers and eGFP protein expression were measured by flow cytometry on a BD LSRFortessa (BD Biosciences). In addition, translation efficiency of eGFP-encoding mRNA was analyzed by polysome profiling as previously described in Damgaard and Lykke-Andersen (2011). Total, cytoplasmic, and nuclear RNA levels as well as the influence of WPRE on nuclear RNA export were evaluated by both eGFP-specific TaqMan RT-qPCR and RNA-FISH. Finally, colocalization between TuD transcripts and P-bodies was evaluated by combined FISH using eGFP-specific probes and immunostaining using hDcpla antibodies.

\section{SUPPLEMENTAL MATERIAL}

Supplemental material is available for this article.

\section{ACKNOWLEDGMENTS}

We thank Lisbeth Dahl Schrøder for technical assistance. This work was made possible through support from the Lundbeck Foundation, Aase and Ejnar Danielsen Foundation, Agnes and Poul Friis Foundation, Carl and Ellen Hertz' grant for Medical and Natural Sciences, Harboe Foundation, Arvid Nilsson Foundation, Foundation of 1870, Direktør Emil C. Hertz og hustru Inger Hertz' Fond, and Fabrikant Ejnar Willumsens Legat. A.K.H. was enrolled at and funded by the Graduate School of HEALTH, Aarhus University. J.G.M. is the head of Gene Therapy Initiative Aarhus (GTI-Aarhus), funded by the Lundbeck Foundation, and a member of the Aarhus Research Center for Innate Immunology (ARCII), established through funding by the AU-Ideas program at Aarhus University. Flow cytometry and cell sorting were performed at the FACS Core Facility, Aarhus University, Denmark.

Received March 28, 2017; accepted April 26, 2017.

\section{REFERENCES}

Bak RO, Mikkelsen JG. 2014. miRNA sponges: soaking up miRNAs for regulation of gene expression. Wiley Interdiscip Rev RNA 5: 317-333.
Bak RO, Hollensen AK, Mikkelsen JG. 2013a. Managing microRNAs with vector-encoded decoy-type inhibitors. Mol Ther 21: $1478-1485$.

Bak RO, Hollensen AK, Primo MN, Sørensen CD, Mikkelsen JG. 2013b. Potent microRNA suppression by RNA Pol II-transcribed 'Tough Decoy' inhibitors. RNA 19: 280-293.

Damgaard CK, Lykke-Andersen J. 2011. Translational coregulation of 5'TOP mRNAs by TIA-1 and TIAR. Genes Dev 25: 2057-2068.

Decker CJ, Parker R. 2012. P-bodies and stress granules: possible roles in the control of translation and mRNA degradation. Cold Spring Harb Perspect Biol 4: a012286.

Ding L, Spencer A, Morita K, Han M. 2005. The developmental timing regulator AIN-1 interacts with miRISCs and may target the Argonaute protein ALG-1 to cytoplasmic P bodies in C. elegans. Mol Cell 19: 437-447.

Donello JE, Beeche AA, Smith GJ III, Lucero GR, Hope TJ. 1996. The hepatitis $B$ virus posttranscriptional regulatory element is composed of two subelements. J Virol 70: 4345-4351.

Donello JE, Loeb JE, Hope TJ. 1998. Woodchuck hepatitis virus contains a tripartite posttranscriptional regulatory element. J Virol 72: 5085-5092.

Ebert MS, Sharp PA. 2010. MicroRNA sponges: progress and possibilities. RNA 16: 2043-2050.

Eystathioy T, Jakymiw A, Chan EK, Séraphin B, Cougot N, Fritzler MJ. 2003. The GW182 protein colocalizes with mRNA degradation associated proteins hDcpl and hLSm4 in cytoplasmic GW bodies. RNA 9: $1171-1173$.

Fabian MR, Sonenberg N, Filipowicz W. 2010. Regulation of mRNA translation and stability by microRNAs. Annu Rev Biochem 79: 351-379.

Franks TM, Lykke-Andersen J. 2008. The control of mRNA decapping and P-body formation. Mol Cell 32: 605-615.

Gentner B, Schira G, Giustacchini A, Amendola M, Brown BD, Ponzoni M, Naldini L. 2009. Stable knockdown of microRNA in vivo by lentiviral vectors. Nat Methods 6: 63-66.

Haraguchi T, Ozaki Y, Iba H. 2009. Vectors expressing efficient RNA decoys achieve the long-term suppression of specific microRNA activity in mammalian cells. Nucleic Acids Res 37: e43.

Haraguchi T, Nakano H, Tagawa T, Ohki T, Ueno Y, Yoshida T, Iba H. 2012. A potent $2^{\prime}$-O-methylated RNA-based microRNA inhibitor with unique secondary structures. Nucleic Acids Res 40: e58.

Higashimoto T, Urbinati F, Perumbeti A, Jiang G, Zarzuela A, Chang LJ, Kohn DB, Malik P. 2007. The woodchuck hepatitis virus post-transcriptional regulatory element reduces readthrough transcription from retroviral vectors. Gene Ther 14: 1298-1304.

Höhne M, Schaefer S, Seifer M, Feitelson MA, Paul D, Gerlich WH. 1990. Malignant transformation of immortalized transgenic hepatocytes after transfection with hepatitis B virus DNA. EMBO J 9: 1137-1145.

Hollensen AK, Bak RO, Haslund D, Mikkelsen JG. 2013. Suppression of microRNAs by dual-targeting and clustered Tough Decoy inhibitors. RNA Biol 10: 406-414.

Jakobsen M, Stenderup K, Rosada C, Moldt B, Kamp S, Dam TN, Jensen TG, Mikkelsen JG. 2009. Amelioration of psoriasis by antiTNF- $\alpha$ RNAi in the xenograft transplantation model. Mol Ther 17: 1743-1753.

Janssen HL, Reesink HW, Lawitz EJ, Zeuzem S, Rodriguez-Torres M, Patel K, van der Meer AJ, Patick AK, Chen A, Zhou Y, et al. 2013. Treatment of HCV infection by targeting microRNA. $N$ Engl $J$ Med 368: 1685-1694.

Kingsman SM, Mitrophanous K, Olsen JC. 2005. Potential oncogene activity of the woodchuck hepatitis post-transcriptional regulatory element (WPRE). Gene Ther 12: 3-4.

Klein R, Ruttkowski B, Knapp E, Salmons B, Gunzburg WH, Hohenadl C. 2006. WPRE-mediated enhancement of gene expression is promoter and cell line specific. Gene 372: 153-161.

Krützfeldt J, Rajewsky N, Braich R, Rajeev KG, Tuschl T, Manoharan M, Stoffel M. 2005. Silencing of microRNAs in vivo with 'antagomirs'. Nature 438: 685-689. 
Loeb JE, Cordier WS, Harris ME, Weitzman MD, Hope TJ. 1999. Enhanced expression of transgenes from adeno-associated virus vectors with the woodchuck hepatitis virus posttranscriptional regulatory element: implications for gene therapy. Hum Gene Ther 10: 2295-2305.

Lu Z, Li Y, Takwi A, Li B, Zhang J, Conklin DJ, Young KH, Martin R, Li Y. 2011. miR-301a as an NF- $\kappa B$ activator in pancreatic cancer cells. EMBO J 30: 57-67.

Mastroyiannopoulos NP, Feldman ML, Uney JB, Mahadevan MS, Phylactou LA. 2005. Woodchuck post-transcriptional element induces nuclear export of myotonic dystrophy $3^{\prime}$ untranslated region transcripts. EMBO Rep 6: 458-463.

McDermott AM, Heneghan HM, Miller N, Kerin MJ. 2011. The therapeutic potential of microRNAs: disease modulators and drug targets. Pharm Res 28: 3016-3029.

Michler T, Große S, Mockenhaupt S, Röder N, Stückler F, Knapp B, Ko C, Heikenwalder M, Protzer U, Grimm D. 2016. Blocking sense-strand activity improves potency, safety and specificity of anti-hepatitis B virus short hairpin RNA. EMBO Mol Med 8: 1082-1098.

Mockenhaupt S, Grosse S, Rupp D, Bartenschlager R, Grimm D. 2015. Alleviation of off-target effects from vector-encoded shRNAs via codelivered RNA decoys. Proc Natl Acad Sci 112: E4007-E4016.

Parker R, Sheth U. 2007. P bodies and the control of mRNA translation and degradation. Mol Cell 25: 635-646.

Popa I, Harris ME, Donello JE, Hope TJ. 2002. CRM1-dependent function of a cis-acting RNA export element. Mol Cell Biol 22: 2057-2067.

Primo MN, Bak RO, Schibler B, Mikkelsen JG. 2012. Regulation of proinflammatory cytokines TNFa and IL24 by microRNA-203 in primary keratinocytes. Cytokine 60: 741-748.

Qiu H, Zhong J, Luo L, Liu N, Kang K, Qu J, Peng W, Gou D. 2015. A PCR-based method to construct lentiviral vector expressing double tough decoy for miRNA inhibition. PLoS One 10: e0143864.

Sakurai F, Furukawa N, Higuchi M, Okamoto S, Ono K, Yoshida T, Kondoh M, Yagi K, Sakamoto N, Katayama K, et al. 2012. Suppression of hepatitis $\mathrm{C}$ virus replicon by adenovirus vector-mediated expression of tough decoy RNA against miR-122a. Virus Res 165: 214-218.

Salmon P, Kindler V, Ducrey O, Chapuis B, Zubler RH, Trono D. 2000. High-level transgene expression in human hematopoietic progenitors and differentiated blood lineages after transduction with improved lentiviral vectors. Blood 96: 3392-3398.

Schambach A, Wodrich H, Hildinger M, Bohne J, Kräusslich HG, Baum C. 2000. Context dependence of different modules for post- transcriptional enhancement of gene expression from retroviral vectors. Mol Ther 2: 435-445.

Schambach A, Bohne J, Baum C, Hermann FG, Egerer L, von Laer D, Giroglou T. 2006. Woodchuck hepatitis virus post-transcriptional regulatory element deleted from $\mathrm{X}$ protein and promoter sequences enhances retroviral vector titer and expression. Gene Ther 13: 641-645.

Schambach A, Galla M, Maetzig T, Loew R, Baum C. 2007. Improving transcriptional termination of self-inactivating $\gamma$-retroviral and lentiviral vectors. Mol Ther 15: 1167-1173.

Scherr M, Venturini L, Battmer K, Schaller-Schoenitz M, Schaefer D, Dallmann I, Ganser A, Eder M. 2007. Lentivirus-mediated antagomir expression for specific inhibition of miRNA function. Nucleic Acids Res 35: e149.

Sen GL, Wehrman TS, Blau HM. 2005. mRNA translation is not a prerequisite for small interfering RNA-mediated mRNA cleavage. Differentiation 73: 287-293.

van der Ree MH, van der Meer AJ, de Bruijne J, Maan R, van Vliet A, Welzel TM, Zeuzem S, Lawitz EJ, Rodriguez-Torres $M$, Kupcova V, et al. 2014. Long-term safety and efficacy of microRNA-targeted therapy in chronic hepatitis C patients. Antiviral Res 111: 53-59.

Wei Y, Etiemble J, Fourel G, Vitvitski-Trepo L, Buendia MA. 1995. Hepadna virus integration generates virus-cell cotranscripts carrying $3^{\prime}$ truncated X genes in human and woodchuck liver tumors. J Med Virol 45: 82-90.

Winter J, Jung S, Keller S, Gregory RI, Diederichs S. 2009. Many roads to maturity: microRNA biogenesis pathways and their regulation. Nat Cell Biol 11: 228-234.

Xie J, Ameres SL, Friedline R, Hung JH, Zhang Y, Xie Q, Zhong L, Su Q, He R, Li M, et al. 2012. Long-term, efficient inhibition of microRNA function in mice using rAAV vectors. Nat Methods 9: 403-409.

Xu ZL, Mizuguchi H, Mayumi T, Hayakawa T. 2003. Woodchuck hepatitis virus post-transcriptional regulation element enhances transgene expression from adenovirus vectors. Biochim Biophys Acta 1621: 266-271.

Zanta-Boussif MA, Charrier S, Brice-Ouzet A, Martin S, Opolon P, Thrasher AJ, Hope TJ, Galy A. 2009. Validation of a mutated PRE sequence allowing high and sustained transgene expression while abrogating WHV-X protein synthesis: application to the gene therapy of WAS. Gene Ther 16: 605-619.

Zufferey R, Donello JE, Trono D, Hope TJ. 1999. Woodchuck hepatitis virus posttranscriptional regulatory element enhances expression of transgenes delivered by retroviral vectors. J Virol 73: 2886-2892. 

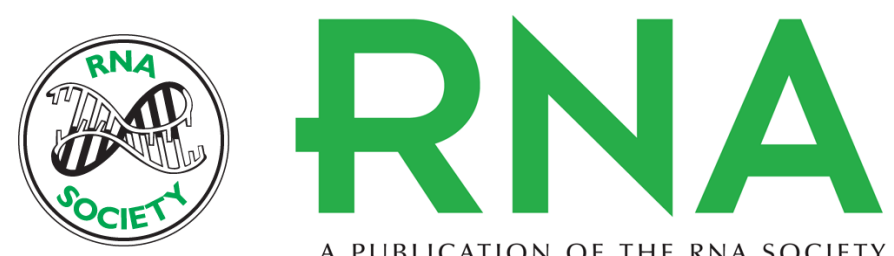

A PUBLICATION OF THE RNA SOCIETY

\section{Improved microRNA suppression by WPRE-linked tough decoy microRNA sponges}

Anne Kruse Hollensen, Rune Thomsen, Rasmus O. Bak, et al.

RNA 2017 23: 1247-1258 originally published online May 9, 2017

Access the most recent version at doi:10.1261/rna.061192.117

\section{Supplemental http://rnajournal.cshlp.org/content/suppl/2017/05/09/rna.061192.117.DC1 Material}

References This article cites 47 articles, 14 of which can be accessed free at: http://rnajournal.cshlp.org/content/23/8/1247.full.html\#ref-list-1

Creative This article is distributed exclusively by the RNA Society for the first 12 months after the Commons License full-issue publication date (see http://rnajournal.cshlp.org/site/misc/terms.xhtml). After 12 months, it is available under a Creative Commons License (Attribution-NonCommercial 4.0 International), as described at http://creativecommons.org/licenses/by-nc/4.0/.
Email Alerting Receive free email alerts when new articles cite this article - sign up in the box at the Service top right corner of the article or click here.

\section{|||||||| Providing Precise Solutions for your research.}

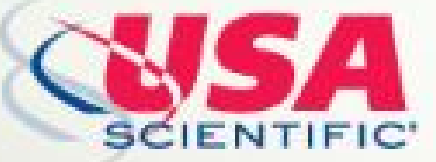

To subscribe to $R N A$ go to:

http://rnajournal.cshlp.org/subscriptions

(C) 2017 Hollensen et al.; Published by Cold Spring Harbor Laboratory Press for the RNA Society 